Editorial

\title{
Acknowledgment to Reviewers of Algorithms in 2020
}

\author{
Algorithms Editorial Office
}

Citation: Algorithms Editorial Office. Acknowledgment to Reviewers of Algorithms in 2020. Algorithms 2021, 14, 33. https://doi.org/10.3390/a1402 0033

Published: 25 January 2021

Publisher's Note: MDPI stays neutral with regard to jurisdictional claims in published maps and institutional affiliations.

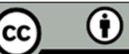

Copyright: (c) 2021 by the author. Licensee MDPI, Basel, Switzerland. This article is an open access article distributed under the terms and conditions of the Creative Commons Attribution (CC BY) license (http://creativecommons.org/licenses /by/4.0/).

MDPI AG, St. Alban-Anlage 66, 4052 Basel, Switzerland

Peer review is the driving force of journal development, and reviewers are gatekeepers who ensure that Algorithms maintains its standards for the high quality of its published papers. Thanks to the cooperation of our reviewers, in 2020, the median time to first decision was 17 days and the median time to publication was 40 days. The editors would like to express their sincere gratitude to the following reviewers for their precious time and dedication, regardless of whether the papers were finally published:

Abbruzzo, Antonino

Abdalla, Rifaat

Abdellatif, Mohamed

Abreu, António

Adacher, Ludovica

Adhi Tama, Bayu

Alanqar, Anas

Albu, Felix

Alexan, Wassim

Alger, Nick

Al-Gumaei, Yousef

Ali, Sajid

Alicea, Bradly

Allouche, David

Almeida Mendes, Mateus Daniel

Almeida, Thiago

Alonso, Miguel A.

Al-qaness, Mohammed A. A.

Al-Rifaie, Mohammad Majid

Al-Sadoon, Mohammed

Alsharif, Mohammed H.

Amancio, Diego

Amaral, Marco Antonio

Amezquita Sanchez, Juan Pablo

Amin, Sikandar

Amirghasemi, Mehrdad

Amirshahi, Seyed Ali

Andronache, Ion C.

Anselm, Blumer

Antunes, Mário

Apruzzese, Giovanni

Aranda-Corral, Gonzalo A.

Aryal, Jagannath

Asensi, Adrià

Aslam, Muhammad
Aßmus, Marcus

Audrito, Giorgio

Augusto, Adriano

Ávalos, Juan Gerardo

Azam, Naveed Ahmed

Babii, Andrii

Bacolla, Albino

Baek, Joong-Hwan

Bai, Song

Baioletti, Marco

Baker, Thar

Balado Frías, Jesús

Balaras, Constantinos A.

Bannach, Max

Baptista, Marcia

Barabino, Benedetto

Baranovskii, Evgenii S.

Barbosa, Ramiro S.

Barbucha, Dariusz

Barile, Sergio

Barthelme, Simon

Barton, Michael

Basak, Sanchita

Basan, Elena

Basler, Georg

Bautu, Elena

Bawazeer, Saleh A.

Bazighifan, Omar

Bazydło, Grzegorz

Beham, Andreas

Belzunce, Martin

Benítez, Rafael

Bentes, Carlos

Bergadano, Francesco

Berhausen, Sebastian 
Berlanga, Rafael

Berlizov, Andrey

Berndt, Sebastian

Bernhauerová, Veronika

Berthouze, Luc

Bhuiyan, Ehsan

Bianchi, Filippo Maria

Bianconi, Francesco

Bigand, Andre

Bille, Philip

Bilò, Davide

Bilò, Vittorio

Bingert, Sven

Björklund, Henrik

Bobillo, Fernando

Bodyanskiy, Yevgeniy

Boettcher, Stefan

Bolboacă, Sorana D.

Bonomo, Flavia

Borkowski, Piotr

Borowczyk, Henryk

Borrego-Díaz, Joaquin

Borunda, Mónica

Bota, Monica

Botnan, Magnus Bakke

Botzheim, Janos

Boubchir, Larbi

Boudjadar, Jalil

Bourva, Ludovic

Brandt, Steven R.

Brandusoiu, Ionut Bogdan

Brcic, Mario

Bréhier, Charles-Edouard

Brkic, Dejan

Browne, Ryan

Brownlee, Alexander

Bruno, Rodrigo

Buchmeister, Borut

Bugajev, Andrej

Burdett, Robert

Burinskienè, Aurelija

Burrascano, Pietro

Butenkov, Sergey A.

Calbimonte, Jean-Paul

Calegari, Roberta

Callejón-Ferre, Ángel-Jesús

Campi, Cristina

Campos Souza, Paulo Vitor

Candelas-Herias, Francisco Andres

Candido, Renato

Cang, Zixuan
Cao, Zhixing

Cara, Enrique Fernández

Carbone, Anna

Carden, Steve

Carrière, Mathieu

Carutasiu, Mihail-Bogdan

Carvalho, Micael Cabrera

Casalino, Gabriella

Castellanos Garzón, José Antonio

Castillejo Parrilla, Pedro

Cavique, Luís

Ceccacci, Silvia

Ceccarello, Matteo

Celińska-Janowicz, Dorota

Cerello, Piergiorgio

Čereška, Audrius

Chadjipandelis, Theodoros

Chadwick, Ruth

Chakraborty, Basabi

Chandrasekaran, Srinivas Niranj

Chang, Ching-Lueh

Chatterjee, Ayan

Chen, Diyi

Chen, Kuen-Suan

Chen, Raymond

Chen, Tinggui

Chernyi, Sergei

Chicharro, Francisco

Chicone, Carmen

Chih, Mingchang

Chiu, Christopher

Cho, Younggun

Choi, Jaeyoung

Choi, Sungyun

Choi, Tsan-Ming

Chong, Edwin K. P.

Choudhury, Farhana

Christofides, Panagiotis

$\mathrm{Chu}$, Eric King-Wah

Chuang, Yen-Ching

CHUI, Kwok Tai

Chwastek, Krzysztof

Cichosz, Paweł

Ciecholewski, Marcin

Cire, Andre Augusto

Civera, Marco

Cleghorn, Christopher W

Cockshott, William

Constantinides, Anthony G.

Conte, Alessio

Corizzo, Roberto 
Costanza, Pascal

Costa-Requena, Jose

Costin, Aaron

Cota, Luciano Perdigão

Craus, Mitica

Crippa, Paolo

Crochemore, Maxime

Cuevas De La Rosa, Francisco

D'Acierno, Luca

D'Andreagiovanni, Fabio

D'Angelo, Gianlorenzo

Dang-Nguyen, Duc-Tien

Daniels, Noah M.

Danilov, Alexander

Dannélls, Dana

Darrah, Marjorie

Dasygenis, Minas

Daunys, Gintautas

David, Sergio

De Amorim, Renato Cordeiro

De Benedictis, Riccardo

De Diego Onsurbe, José Antonio

De La Fraga, Luis G.

De Leone, Renato

De Marchi, Stefano

De Mingo López, Luis Fernando

De Sa, Christopher

De Vito, Danila

Defryn, Christof

Delcea, Camelia

Dell'Oca, Aronne

Demidova, Liliya A.

Denisiuk, Aleksander

Derr, Tyler

Dev, Soumyabrata

Dewasme, Laurent

Di Corso, Evelina

Di Matteo, Alberto

Diaz, Rocio Gonzalez

Dimitrov, Borislav

Ding, Feng

Ding, Shihong

Dinneen, Michael J.

Djeziri, Mohand Arab

Dobrzycki, Arkadiusz

Dochow, Robert

Dolega, Boguslaw

Domínguez, Luis Vergara

Donelli, Massimo

Dorożyński, Przemysław

Dos Santos Azevedo, Juarez
Dósa, György

Dovgan, Erik

Dragisa, Stanujkic

Du, Yegang

Duarte, Denio

Dulebenets, Maxim A.

Duncan, Dominique

Durán Parejo, Francisco

Dutta, Soumya

Dwivedi, Ashutosh Dhar

Dzedzickis, Andrius

Dzido, Tomasz

E. Kvasov, Dmitri

Eberhard, Andrew

Edoh, Kossi

Eduardo Espitia, Helbert

Eftimov, Tome

El Euch, Hichem

Eliáš, Petr

El-Khazali, Reyad

Elmouki, Ilias

Endo, Patricia Takako

Engelbrecht, Andries

Escolar, Emerson G.

Esposito, Antonio

Evans, Michael

Fabelo, Himar

Fan, Shu-Kai S.

Farago, Andras

Farago, Paul

Farias, Delia

Farquharson, Colin

Feld, Sebastian

Fernández Fernández, Francisco Javier

Fernández-Isabel, Alberto

Fernau, Henning

Ferreira, Ricardo

Ferri, Massimo

Feschet, Fabien

Fieno Da Silva, Alexandre

Figueroa, Karina

Fijorek, Kamil

Fiorini, Rodolfo A.

Firšt Rogale, Snježana

Fish, Andrew

Fister, Iztok Jr.

Fletcher, Kenneth

Fonod, Robert

Foraboschi, Paolo

Főző, Ladislav

Fraga, Mariana Amorim 
Francisco, Gómez Aguilar José

Frasca-Caccia, Gianluca

Freiherr Von Lukas, Uwe

Freij-Hollanti, Ragnar

Frigioni, Daniele

Frolov, Nikita

Frontoni, Emanuele

$\mathrm{Fu}$, Cheng

$\mathrm{Fu}$, Tak-chung

Fuchigami, Helio Yochihiro

Fuente, María J.

Fugacci, Ulderico

Fukuda, Atsushi

Furmańczyk, Hanna

Gagie, Travis

Gallegos, Javier

Gallegos-Funes, Francisco

Gao, Junqi

Gao, Shangce

Gao, Wei

García-Loygorri, Juan Moreno

Garg, Harish

Gąska, Adam

Gavrilova, Marina

Genge, Bela

Gerothanassis, Ioannis P.

Ghani, Arfan

Ghayoumi, Mehdi

Ghazi-Nezami, Farnaz

Ghita, Bogdan

Ghoreyshi, Seyed Mohammad

Ghosh, Sayan

Ghysels, Pieter

Giorgio, Gosti

Goda, Takashi

Goedgebeur, Jan

Goldfarb, Boris

Gomes, Guilherme Ferreira

Gómez-Aguilar, José Francisco

González-Gaxiola, Oswaldo

Gorecki, Pawel

Gőring, Frank

Gouiffès, Michèle

Griewank, Andreas

Grimaccia, Francesco

Grime, Geoffrey

Grishagin, Vladimir A.

Gu, Xianming

$\mathrm{Gu}$, Yuantao

Guerra, Maria Letizia

Guo, Baofeng
Guo, Dezhou

Guo, Jianhua

Guo, Xiansheng

Guo, Xue-Ping

Guo, Yanyong

Gupta, Anupam

Gutiérrez, Juan Manuel

Gutt, Robert

Guzmán, Lev

Hadad, Yossi

Haeusler, Edward

Hajij, Mustafa

Hamdaoui, Mohamed

Han, Xiaohui

Haque, Sardar Anisul

Harizanov, Stanislav

Harrod, Steven

Heck, André

Heger, Jens

Heiland, Ines

Hejmanowski, Ryszard

Helbig, Marde

Heni, Hamza

Henriques, João

Heras, Jónathan

Herrán González, Alberto

Higson, Edward

Hildmann, Hanno

Hin, Ooi Ean

Hishinuma, Kazuhiro

Ho, Hong-Xuyen Thi

Hong, Danfeng

Hong, Wei-Chiang

Hooghoudt, Jan-Otto

Horák, Jakub

Horla, Dariusz

Horng, Shih-Cheng

Hosseinzadeh, Mehdi

Hristova, Snezhana

Hsie, Sun-Yuan

Hua, Jie

Huang, Dong

Huang, Jih-Jeng

Huang, Ko-Wei

Huang, Shih-Chang

Ianole-Calin, Rodica

Iliyasu, Abdullah

Imakura, Akira

Ingber, Lester

Ionescu, Tudor C.

Ishaq, Isam 
Islam, Raihan Ul

Ivanov, Stoil I.

Izquierdo, Estibaliz Martinez

Jafari, Mohammad

Jafari, Soheil

Jakubas, Adam

Jan, Chyan-Long

Jang, Han Seung

Janiszewski, Dariusz

Janjua, Naeem

JÄNTSCHI, Lorentz

Jasiulewicz-Kaczmarek, Malgorzata

Jayasumana, Sadeep

Jeon, Jun-Cheol

Jeong, Dong Hyun

Jiang, Fan

Jiang, Shaofeng

Jianu, Radu

Jin, Xin

Jónás, Tamás

Jones, Karl O.

Joshi, Gyanendra P.

Jovanovic, Raka

Juhász, László

Kabir, Sohag

Kajiwara, Yusuke

Kalenkova, Anna

Kalina, Martin

Kamsu-Foguem, Bernard

Kanamori, Takafumi

Kapsalis, Vassilis

Kapuscinski, Tomasz

Karakasidis, Alexandros

Karakatič, Sašo

Karakostas, George

Karapinar, Erdal

Karie, Nickson M.

Kasnesis, Panagiotis

Kataoka, Hirokatsu

Katina, Polinpapilinho F.

Katsikouli, Panagiota

Kawahara, Jun

Kay, Jim W.

Kazakbaev, Vadim

Keler, Andreas

Keriven, Nicolas

Khachay, Michael

Khalaf, Walaa

Khalili Mobarakeh, Ali

Khanh Le, Nguyen Quoc

Khoa, Vo Anh
Khodadadian, Amirreza

Kim, Hak-Seon

Kim, Jon-Lark

Klamt, Steffen

Klanšek, Uroš

Klein, Shmuel Tomi

Kluza, Krzysztof

Knop, Dusan

Knudson, Kevin

Knypiński, Łukasz

Kołakowska, Agata

Kolog, Emmanuel Awuni

Kolomvatsos, Kostas

Kontogiannis, Sotirios

Köppl, Dominik

Korvel, Grazina

Kosolobov, Dmitry

Kotsiantis, Sotiris

Koutras, Thanasis

Kouziokas, Georgios N.

Kovács, Zoltán

Kowalik, Rafał

Kowalski, Gregory

Kravari, Kalliopi

Kröker, Ilja

Kubiak, Ireneusz

Kudryashov, Boris

Kulpa, Krzysztof

Kunkel, Julian

Kuppannagari, Sanmukh R

Kuznetsov, Nikolay V.

Kwon, Joseph Sang-Il

Lacroix, Pierre

Ladaci, Samir

Lafond, Manuel

Lahoz, Rafael

Lange, Julia

Lawnik, Marcin

Lazarescu, Mihai

Le, Nguyen Quoc Khanh

Łebkowski, Andrzej

Lechner, Ulrike

Lecroq, Thierry

Lee, Chou-Yuan

Lee, Eui Chul

Lee, Kwang Y.

Lee, Kyu-haeng

Lee, Posen

Lee, Sang Jun

Lee, Seokcheon

Lee, Seunggyu 
Lee, Seyong

Lekidis, Vassilios

Lekunberri, Edorta Carrascal

Leliaert, Jonathan

Leng, Siyang

Lenk, Peter

Lenzi, Marcelo

Lesniak, Zbigniew

Leszczuk, Mikołaj

Leucci, Stefano

Levina, Alla

Lewis, Rhyd

Li, Chunguo

Li, Taiyong

Li, Weide

Li, Xiangtao

Li, Xiaowu

Li, Xingjie

Li, Zhongyi

Lin, Chaoyuan

Lin, Zhongwei

Lipowski, Adam

Lirkov, Ivan Dimov

Liu, Chang

Liu, Chenglin

Liu, Huafeng

Liu, Qingfeng

Liu, Tong

Liu, Wei-Min

Liu, Yajing

Livieris, Ioannis E.

Lizcano, David

Loddo, Andrea

Loperfido, Nicola

Lopez-Cruz, Irineo

López-Núñez, Alejandro

Lorig, Fabian

Lotfian, Saeid

Lou, Yang (Felix)

Loukrezis, Dimitrios

Louza, Felipe

Loza Mencía, Eneldo

Lu, Min

Lucia, Parussini

Lucille, Alonso

Luo, Hanjiang

Ma, Will

Machura, Lukasz

Maciel, Carlos Dias

Madleňák, Radovan

Madokoro, Hirokazu
Madsen, Chad

Mahmud, M. Rizaludin

Majdara, Aref

Makris, Christos

Malinowska, Agnieszka

Malinowski, Marek T.

Mambretti, Joe

Mancini, Simona

Mandarawi, Waseem

Mandric, Igor

Manis, George

Mantzaris, Alexander V.

Mao, Yao

Marcinkiewicz, Michal

Margaris, Dionisis

Mari, Federico

Mariani, Maria

Marongiu, Jean Christophe

Marović, Ivan

Marques, Antonio Garcia

Marrero, Domingo

Marszalek, Zbigniew

Martínez López, José Israel

Martínez López, Luis

Martino, Luca

Martyshko, Peter S.

Maru, Marcia M.

Marx, Edgard

Maskeliunas, Rytis

Mata Miquel, Christian

Matevossian, Hovik

Matouk, Ahmed Ezzat

Maturo, Fabrizio

Mawlana, Mohammed

Mayer, Joceli

McAlinn, Kenichiro

McKendall, Alan

McPheron, Benjamin D.

Meeks, Kitty

Meidute-Kavaliauskiene, Ieva

Meiguins, Bianchi

Mellin, Patricia

Meloni, Carlo

Mercader, Pedro

Mercer, David J.

Mernik, Marjan

Mielewczik, Michael

Mieloszyk, Magdalena

Migallón, Héctor

Miglionico, Giovanna

Migniot, Cyrille 
Mikayilov, Jeyhun I

Milani, Alfredo

Milenković, Miloš

Milik, Adam

Minghim, Rosane

Mingotti, Alessandro

Misevicius, Alfonsas

Misra, Neeldhara

Mjahed, Mostafa

Mogale, Dnyaneshwar

Mohamed, Ali Wagdy

Mohamed, M Shadi

Molinero, Xavier

Mollica, Cristina

Molnar, Miklos

Monaco, Gianpiero

Monod, Anthea

Montoya, Alejandro

Moon, Jihoon

Moreira, Mário W. L.

Moreno Beltrán, Plácido

Moreno, Jaime

Morozov, Vitaly A.

Mosavi, Amir

Mostafa, Ahmed

Mrozek, Dariusz

Muller, Felipe Martins

Mura, Michela Dalle

Mystkowski, Arkadiusz

Nabil, Mahmoud

Nakano, Mariko

Nakashima, Yuto

Nalepa, Jakub

Naraigh, Lennon O.

Narayanan, Barath

Naser, M. Z.

Nataliya, Dilna

Navara, Mirko

Nepomuceno, Erivelton Geraldo

Nguyen, Van Dung

Niazazari, Iman

Nielsen, Peter

Nivedita, Nivedita

Noeiaghdam, Samad

Norikazu, TAKAHASHI

Novič, Marjana

Ojeda, Dora Luz

Ojsteršek, Robert

Okada, Isamu

Olivares-Benitez, Elias

Oliveira, Helder
Oliveira, Tiago Roux

Olivera, Ana Carolina

Olivieri, Annamaria

Ontanon-Garcia, L.J.

Orosz, Tamás

Osawa, Minoru

Osuna-Enciso, Valentín

Oszust, Mariusz

Ou, Weihua

Ougolnitsky, Guennady

Page, Daniel R.

Pais, Sebastião

Pait, Felipe

Pamucar, Dragan

Pamuła, Teresa

Pandey, Prem Chandra

Panić, Branislav

Papadakos, Panagiotis

Pardo Martínez, Clara Inés

Pardo, J. M.

Parikesit, Arli Aditya

Parsopoulos, Konstantinos

Pascale, Francesco

Pascolo, Paolo

Pătrașcu, Monica

Patrone, Fabio

Pauk, Jolanta

Pavaloaia, Vasile Daniel

Peitz, Sebastian

Pellegrino, Maria Angela

Pelletier, Mathew G.

Peña Pitarch, Esteban

Peretz, Yossi

Pérez De Prado, Rocío Josefina

Pérez, Gustavo

Perne, Matija

Perracchione, Emma

Perraudin, Nathanaël

Perry, Stuart

Petroșanu, Dana-Mihaela

Piasecki, Krzysztof

Piasini, Eugenio

Pirola, Yuri

Pitarch, Jose Luis

Pitas, Charalampos N.

Pittino, Federico

Pohjankukka, Jonne

Połap, Dawid

Polonik, Wolfgang

Poniszewska-Maranda, Aneta

Popkov, Yuri S. 
Portugal, Renato

Possenti, Luca

Posth, Jan-Alexander

Pouliquen, Mathieu

Pozo, Francesc

Prandtstetter, Matthias

Prasad, Dilip K.

Pratas, Diogo

Pražák, Dalibor

Price, Christopher John

Proietti, Guido

Providas, E.

Przybyszewski, Andrzej

Psaila, Giuseppe

Pupyrev, Sergey

Pushkarev, Pavel

Qasha, Rawaa

Qi, Guanqiu

Qu, Qiang

Quessy, Jean-François

Rabe, Markus

Rabelo, Luis

Radac, Mircea-Bogdan

Radenovic, Stojan

Radescu, Radu

Rahman, Raziur

Rajabi, Enayat

Rakhmangulov, Aleksandr

Ramos-López, Darío

Rana, Abu Ul Hassan Sarwar

Rasche, Christoph

Recio, Tomas

Regmi, Samundra

Remolar Quintana, Inmaculada

Rempala, Grzegorz

Revetria, Roberto

Revinova, Svetlana

Reyes Menéndez, Ana

Rezaei Rad, Aryan

Rezapour Mashhadi, Mohammad Mahdi

Riba Ruiz, Jordi-Roger

Ribeiro, Tiago

Ricaud, Benjamin

Righi, Rodrigo

Rinaldi, Claudia

Rinaldi, Francesco

Rivera, Jorge

Rivero-Angeles, Mario Eduardo

Rizun, Nina

Röbenack, Klaus

Rocha Silva, Rodrigo
Rodríguez, Adrián Fernández

Rodríguez-Licea, Martín-A.

Rodriguez-Resendiz, Juvenal

Rogerio Pinheiro, Plácido

Rogowski, Krzysztof

Rohacs, Daniel

Romaszewski, Michał

Romeo, Luca

Romero-Conrado, Alfonso R.

Rosário, Denis

Rossini, Luca

Rossiter, Anthony

Rueda Ruiz, Antonio Jesús

Ruiz, Oscar

Rundo, Francesco

Rupnik Poklukar, Darja

Rydel, Marek

Sabo, Cosmin

Saborido, Rubén

Sadek, Rowayda A.

Sadollah, Ali

Saeed, Nagham

Safari, Nima

Safonov, Ilia

Sahin, Bekir

Saiko, Guennadi

Sajavičius, Svajūnas

Sałabun, Wojciech

Salamanca, Juan Jesús

Saleh, Adel

Salehi, Saeed

Salido Tercero, Jesús

Salimi, Mehdi

Salmela, Leena

Salvagnin, Domenico

Samuel, John

Sanchez, Mauricio A.

Sanchez-Oro Calvo, Jesus

Sanders, Peter

San-Segundo, Rubén

Santos Pinheiro, Rian Gabriel

Santos-Gago, Juan M.

Saracevic, Muzafer H.

Sarantsev, Andrey

Sato, Takao

Sattar, Junaed

Sauer, Nathalie

Saupe, Dietmar

Saura Lacárcel, José Ramón

Saurabh, Saket

Sawik, Bartosz 
Sayood, Khalid

Schaeffer, Elisa

Schaum, Alexander

Schmidt, Helmut

Schmitz, Gunnar

Schönberger, Jörn

Schroeder, Pascal

Schwiegelshohn, Chris

Scuro, Carmelo

Segall, Richard

Sejong, Oh

Semwal, Monika

Senderovich, Arik

Sengupta, Saptarshi

Serani, Andrea

Seyfired, Armin

Sgora, Aggeliki

Shabbir, Noman

Shadloo, Mostafa S.

Shafiq, Omair

Shakhno, Stepan

Shang, Yilun

Sharma, Gokarna

Sharma, Manik

Shen, Guangrong

Shevgunov, Timofey

Shi, Rongye

Shirobokov, Maksim

Shiue, Yeou-Ren

Shuman, David

Shur, Arseny

Siaulys, Jonas

Sicilia-Montalvo, Juan Antonio

Sidorowicz, Elzbieta

Silva, Arlindo

Silva, Fernando S.

Simone, Gabriele

Singh, Pankaj K

Sioma, Andrzej

Skiba, Marta

Škraba, Andrej

Smarra, Francesco

Sofronov, Georgy

Solteiro Pires, Eduardo José

Sotskov, Yuri

Sotskov, Yuri N.

Spoladore, Daniele

Sprungk, Björn

Sreckovic, Vladimir A.

Srinivasan, Kathiravan

Stefanini, Luciano
Steffen, Eckhard

Steinbach, Marc

Stephenson, Mary C

Stępień, Grzegorz

Stich, Sebastian U.

Stocchero, Matteo

Stoean, Catalin

Strawn, Nate

Streif, Stefan

Strumberger, Ivana

Stübinger, Johannes

Subasi, Abdulhamit

Sucar, Enrique

Sun, Daniel(Jian)

Sun, Jian-Qiao

Sun, LiBo

Sun, Yan

Sun, Yuan

Syafrudin, Muhammad

Syropoulos, Apostolos

Szabo, Alexandre

Szabó, Szilárd

Szajowski, Krzysztof

Szczepaniak, Piotr S.

Szénási, Sándor

Szmelter-Jarosz, Agnieszka

Taghizadeh-Mehrjardi, Ruhollah

Tahmasebi, Pejman

Tamura, Hiroki

Tanackov, Ilija

Tang, Wenzhe

Tang, Yifa

Tang, Yuxing

Tanna, Dushyant

Tarhio, Jorma

Tarits, Pascal

Tayeb, Shahab

Teixeira, Marco Antonio

Teramoto, Atsushi

Thibaut, Vidal

Tiels, Koen

Tlelo-Cuautle, Esteban

Toews, Matthew

Tordeux, Antoine

Torres, Pedro M. B.

Torrisi, Mariano

Touryan, Jonathan

Trautmann, Heike

Tronci, Stefania

Trzepiecinski, Tomasz

Tsionas, Mike 
Tynda, Aleksandr

Tyshchenko, Oleksii

Uchroński, Mariusz

Ullrich, Torsten

Urasaki, Naomitsu

Usarek, Zbigniew

Usbeck, Ricardo

Vaccaro, Maria Grazia

Vacik, Harald

Vakhania, Nodari

Valerga Puerta, Ana Pilar

Valiente, David

Van Bommel, Christopher Martin

Van Der Hurk, Evelien

Varga, Laszló

Vaščák, Ján

Vasilakos, Athanasios

Vasileva, Maria

Vathy-Fogarassy, Ágnes

Veeraraghavan, Prakash

Vega, Pastora

Vejdemo Johansson, Mikael

Veli, Mäkinen

Vicario, Saverio

Vieira, Alex Borges

Viennot, Laurent

Vieru, Dumitru

Villagra, Marcos

Vinci, Cosimo

Virgala, Ivan

Vitelli, Valeria

Vlase, Sorin

Volkau, Ihar

Volkov, Mikhail

Volosencu, Constantin

Vulkov, Lubin G.

Waibel, Christoph

Walas, Krzysztof

Wan, Shaohua

Wang, Gai-Ge

Wang, Ming-Shyan

Wang, Ning

Wang, Pi-Chung

Wang, Shuai

Wang, Yang

Watrobski, Jaroslaw

Way, Michael

Wei, Yiheng

Weigel, Martin

Weitschek, Emanuel

Wensing, Patrick
Werner, Piotr A.

Westerholt, René

Wielgosz, Maciej

Wojciechowski, Pawel

Wolf, Guy

Wolniak, Radosław

Woo, Wai Lok

$\mathrm{Wu}$, Chensheng

Wu, Jianhua

Wu, John

$\mathrm{Wu}$, Jong-Wuu

$\mathrm{Wu}$, Shiqian

$\mathrm{Wu}$, Tailin

Wysocki, Marian

Xia, Jingbo

Xiang, Shiming

Xin, Baogui

Xiong, Qingrong

Yan, Bo

Yan, Liang

Yang, Hee-Deok

Yang, Jingyu

Yang, Liping

Yang, Shichun

Yazdanpanah, Hamed

Yeh, Sheng Lih

Yemelyanov, Vitaliy A.

Yin, Zhaoxia

Yokose, Satoshi

Yoo, Byungseok

Yordanova, Kristina

$\mathrm{Yu}$, Liangjiang

Yu, Mei

$\mathrm{Yu}$, Yang

Zagajewski, Bogdan

Zagan, Ionel

Zama, Fabiana

Zambrano-Martinez, Jorge Luis

Zambrano-Serrano, Ernesto

Zarrin, Javad

Zelst, Sebastiaan Van

Zeng, Guoqiang

Zeydan, Engin

Zhang, Chengzhi

Zhang, Guannan

Zhang, Jianpeng

Zhang, Junling

Zhang, Zhen

Zhao, Xu-dong

Zhou, Lin

Zhou, MengChu 
Zhou, Rong

Zhu, Binhai

Zhu, Yangzhi

$\mathrm{Zhu}, \mathrm{Yi}$

Žilinskas, Antanas
Zolotukhin, Mikhail

Zou, Yajie

Žurauskas, Mantas

Zurita, Grover 